\title{
Peripheral neurological involvement in Shiga toxin- producing Escherichia coli-hemolytic uremic syndrome (STEC-HUS) as a rare complication with a positive outcome in two consecutive children - Case Report
}

\section{Luisa Santangelo}

University of Bari: Universita degli Studi di Bari Aldo Moro

Giuseppe Stefano Netti ( $\nabla$ giuseppestefano.netti@unifg.it)

University of Foggia: Universita degli Studi di Foggia https://orcid.org/0000-0003-3495-2707

\section{Diletta Domenica Torres}

University of Bari: Universita degli Studi di Bari Aldo Moro

Giovanni Piscopo

University of Bari: Universita degli Studi di Bari Aldo Moro

Vincenza Carbone

University of Bari: Universita degli Studi di Bari Aldo Moro

Luciana Losito

IRCCS Medea: Istituto di Ricovero e Cura a Carattere Scientifico Eugenio Medea

Leonardo Milella

University of Bari: Universita degli Studi di Bari Aldo Moro

Maria Luigia Lasorella

University of Bari: Universita degli Studi di Bari Aldo Moro

Pasquale Conti

University of Bari: Universita degli Studi di Bari Aldo Moro

Delio Gagliardi

University of Bari: Universita degli Studi di Bari Aldo Moro

\section{Maria Chironna}

University of Bari: Universita degli Studi di Bari Aldo Moro

\section{Elena Bresin}

Mario Negri Institute for Pharmacological Research: Istituto di Ricerche Farmacologiche Mario Negri

Antonio Trabacca

IRCCS Medea: Istituto di Ricovero e Cura a Carattere Scientifico Eugenio Medea

\section{Elena Ranieri}

University of Foggia: Universita degli Studi di Foggia 


\section{Mario Giordano}

University of Bari: Universita degli Studi di Bari Aldo Moro

\section{Case report}

Keywords: Hemolytic uremic syndrome, Shiga toxin-producing Escherichia, Peripheral nervous system, Eculizumab, Neurorehabilitation, Case report

Posted Date: February 26th, 2021

DOl: https://doi.org/10.21203/rs.3.rs-242540/v1

License: (c) (i) This work is licensed under a Creative Commons Attribution 4.0 International License. Read Full License 


\section{Abstract}

Background. The Neurological involvement is the most common extra-renal complication of Shiga toxinproducing Escherichia coli-hemolytic uremic syndrome (STEC-HUS). On brain magnetic resonance examination, main neurological signs encompass acute lesions of the basal ganglia and the white matter, which could usually regress after Eculizumab infusion. In contrast, STEC-HUS-related peripheral nervous system (PNS) involvement is very rare and, when occurring, it requires a careful management of neurological sequelae and an intensive multidisciplinary neuro-rehabilitation program.

Case presentation. Here, we present two cases of severe and complicated STEC-HUS patients with PNSinvolvement who successfully required therapeutic treatment and an intensive multidisciplinary neurorehabilitation program.

In both cases, PNS-involvement followed the recovery from STEC-HUS-related severe central neurological damage and manifested mainly with marked bilateral motor deficit and hyporeflexia/areflexia in the lower limbs. The peripheral polyneuropathy was treated with immune-suppressive therapy (methylprednisolone pulses, i.v. immunoglobulins, therapeutic plasma exchange), followed by a prolonged intensive neuro-rehabilitation program. After 8 months of neuro-rehabilitation, both patients gained complete functional recovery.

Conclusions. PNS involvement during STEC-HUS is a rare event and potentially leading to severe disability. A timely clinical assessment is mandatory to set up a prompt therapeutic and neurorehabilitation program and to obtain a complete clinical and functional recovery.

\section{Background}

The hemolytic uremic syndrome (HUS) is a well-known but rare disease characterized by microangiopathic hemolytic anemia, thrombocytopenia, and organ damage, often renal dysfunction, which occurs both in adults and in children. The "typical" form is mediated by Shiga-like toxin-producing Escherichia coli (STEC-HUS) or, less commonly, by Shiga toxin-producing Shigella dysenteriae type 1 and Streptococcus Pneumoniae $\left({ }^{1}\right)$. All other causes of HUS have traditionally been referred to as "atypical" (aHUS) in which main cases are due to dysregulation and over-activation of the alternative complement pathway $(2,3,4)$, secondary to a gene mutation or, rarely, to acquired autoantibodies neutralizing some complement system components (e.g. anti-factor $\mathrm{H}$ antibodies) $\left(^{5}\right)$. Rarely, clinical conditions, such as autoimmune diseases, transplantation, cancer, infectious diseases, pregnancy, or use of certain cytotoxic drugs, are associated with secondary forms of HUS $\left({ }^{6}\right)$. In STEC-HUS, the Shiga-like toxin release follows the bloody diarrhea by Entero-Hemorrhagic Escherichia Coli (EHEC). The latter can cause systemic endothelial damage and thrombotic microangiopathy (TMA), which leads to the onset of the classic symptomatic triad (anemia, low platelet count and acute kidney injury). In severe forms of TMA with the involvement of the central nervous system (CNS), STEC-HUS-related morbidity and mortality significantly increase $\left({ }^{7}\right)$. Neurologic complications are the most common extra-renal manifestations in STEC-HUS, 
accounting $20-25 \%$ of STEC-HUS patients. Due to these severe forms, patients are at elevated risk of the worst outcome or serious long-term disability after the acute phase of the disease $(8,9,10,11)$. Peripheral nervous system (PNS) involvement during STEC-HUS instead is very rare and limited cases are reported $\left({ }^{12}\right)$. At now, the key role of the complement system dysregulation is well known in the atypical HUS, while rising evidence underlies its involvement also in the pathogenesis of STEC-HUS $\left({ }^{13}\right)$, thus supporting the "off label" use of an anti-C5-convertase monoclonal antibody (Eculizumab) for treating more severe forms of STEC-HUS (mainly with neurological involvement) $(14,15,16,17)$. In this report, we describe two cases of young female patients (9 and 2-year-old), who developed a serious form of STEC-HUS complicated by severe CNS damage, successfully treated with Eculizumab. They were successively affected by a severely disabling peripheral neurological involvement requiring an intensive multidisciplinary neuro-rehabilitation program at hospital discharge.

\section{Case Presentation}

\section{Case 1}

A previously healthy 9-years-old healthy girl was admitted to the Pediatric Infectious Disease Unit (PIDU) with bloody diarrhea and anemia. According to regional guidelines for bloody diarrhea $\left({ }^{18}\right)$, stool research for STEC was performed and the presence of E. Coli 0111 with gene toxin eae was detected. On day 3 , due to the rapid decline of renal function tests (serum Creatinine or $\mathrm{sCr} 0,94 \mathrm{mg} / \mathrm{dL}$, azotemia $67 \mathrm{mg} / \mathrm{dL}$ ) and platelet (PLT) count $\left(130 \times 10^{3} / \mu \mathrm{L}\right)$ and rising of $\mathrm{LDH}(830 \mathrm{U} / \mathrm{I})$, the patient was transferred to our Pediatric Nephrology Unit. The general conditions were poor and the laboratory parameters further worsened due to the onset of septic shock (sCr $2.74 \mathrm{mg} / \mathrm{dL}$; azotemia $125 \mathrm{mg} / \mathrm{dL}$; WBC $31.2 \times 10^{3} / \mu \mathrm{L}$;

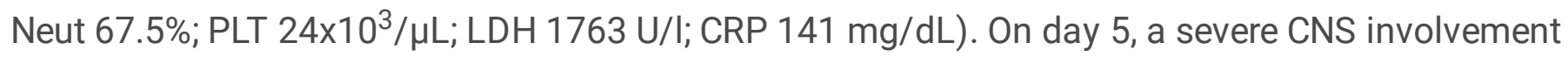
appeared with sudden onset of generalized paresthesia, tingling of the lower limbs, convergence of the eyes, and short but frequent absence seizures ( $<1$ minute of duration). Despite a negative brain computed tomography ( $\mathrm{bCT}$ ) and therapy with benzodiazepines and phenobarbital, at day 6 the general conditions further worsened and a coma status arose (Glasgow Coma Score or GCS 8/15) with immediate transfer to the Pediatric Intensive Care Unit (PICU) and start of the mechanical ventilation.

The Electroencephalography (EEG) revealed diffuse slow-wave activity with delta oscillations in frontal area, while brain magnetic resonance (bMR) showed diffuse ischemic lesions mainly in basal nuclei but also in the brainstem (pons, medulla oblongata). Due to severe neurological involvement, the patient received an off-label treatment with Eculizumab (300 mg IV) on day 6 and after a week (day 13). Moreover, despite massive IV fluid over-hydration and massive diuretic therapy, she became anuric and renal function tests further worsened ( $\mathrm{sCr} 3.3 \mathrm{mg} / \mathrm{dL}$ ). For these reasons, renal replacement therapy (RRT) by vein-vein hemodiafiltration (HDF) was started at day 7 and repeated every day until day 20 . The improvement of blood pressure control was achieved with amlodipine and ramipril therapy. The general condition improved rapidly, thus on day 7 the patient was extubated and returned to our Nephrology Unit. Then, the patient developed a significant visual impairment with evidence of a papilla with blurred 
margins and hemorrhages along the superior temporal arch in the right eye and cotton exudates at the temporal side of the left eye. These findings were compatible with bilateral retinal vascular occlusion.

Progressively, general condition and diuresis improved, with normalization of laboratory parameters (at

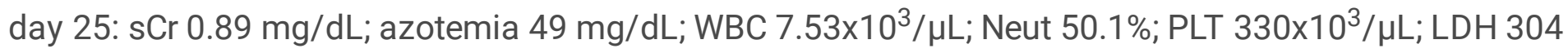
$\mathrm{U} / \mathrm{l} ; \mathrm{CRP} 5 \mathrm{mg} / \mathrm{dL}$ ), so RRT was stopped. Hypokalemia resulting from brisk diuresis was treated with intravenous potassium replacement until it normalized.

A further bMR revealed complete regression of the ischemic hypoxic areas at both the basal nuclei and the brainstem level. Also, EEG and visual impairment were normalized. On day 28, the onset of progressive intense bilateral lower limb pain with marked hyposthenia and inability to keep upright imposed further neurological evaluation. Both spinal cord MR and Cerebrospinal fluid (CSF) analysis were normal. However, electroneurography (ENoG) of peripheral nerves revealed a low amplitude of compound motor action potentials (CMAP) in three sites at the peroneal motor nerve and a reduction of sensory action potential (SAP) at sural sensory nerve bilaterally, thus leading to a diagnosis of inflammatory polyneuropathy (Figure $\mathbf{1 A}-\boldsymbol{B}$ ). Thus, combined therapy with methylprednisolone pulses (10 $\mathrm{mg} / \mathrm{kg} \times 3$ days) and immunoglobulins ( $400 \mathrm{mg} / \mathrm{kg} /$ day for 4 days), followed by a motor-rehabilitation training program, gained a slight and progressive improvement of lower limbs pain and hyposthenia in the child. After 45 days, the patient was transferred to a neuro-rehabilitation center for further intensive treatment. The main clinical and therapeutic information about this case is reported as a timeline in

\section{Figure 2.}

\section{Case 2}

A 2-years-old healthy girl was admitted to PIDU because of bloody diarrhea. Laboratory analysis showed neutrophilic leukocytosis (WBC $13,83 \times 10^{3} / \mu \mathrm{L}$, Neut $67 \%$ ) while other parameters were normal

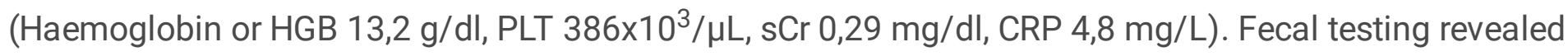
enterohemorrhagic E. coli (STEC 0111) with verocytotoxins Stx2 and eae. Despite IV overhydration and massive diuretic therapy, she developed clinical signs of HUS with anemia, thrombocytopenia $\left(38 \times 10^{3} /\right.$ $\mu \mathrm{L}$ ), and renal function impairment (creatinine $2.28 \mathrm{mg} / \mathrm{dl}$ ) with worsening of general conditions and, successively, oligo-anuria. For these reasons, a session of renal replacement therapy (RRT) by HDF was started. During the first HDF session, the patient showed cardiovascular instability with the onset of neurological signs (eye fixing with strabismus and facial stereotypies). RRT session was suddenly stopped to perform a brain CT scan, resulted normal and then, because of worsening of metabolic acidosis (pH 7.18, $\left.\mathrm{HCO}^{-11} 1.8 \mathrm{mmol} / \mathrm{L}, \mathrm{BE}-15\right)$, the child was moved to PICU. At admission, she showed a coma status (GCS 8/15) and required starting of mechanical ventilation. The neurological assessment revealed normal pupillary reflexes without nuchal rigidity, while EEG revealed diffuse slow-wave activity with theta-delta oscillations of low voltage.

On day 3, due to a condition of severe hyperkalemia resistant to medical therapy (IV calcium gluconate, IV sodium bicarbonate, IV insulin, rectal sodium polystyrene sulfonate), the patient developed life- 
threatening bradycardia requiring successfully cardiopulmonary resuscitation (CPR). Urgently continuous vein-vein HDF was performed over three consecutive days, then it was stopped and replaced by intermittent HDF until day 14. The onset of clinical and laboratory sign of multiorgan failure coupled to rhabdomyolysis required three sessions of CytoSorb ${ }^{\circledR}$ hemoadsorption (Cytosorbents Corporation, Monmouth Junction, NJ, USA) on day 6,8 and $10\left({ }^{19}, 20\right)$.

On day 4, a brain MRI was performed, showing diffuse homogeneous signal hyperintensity in T2 and FLAIR sequences, with evident signal restriction in DWi and homogeneous hypointensity in T1-weighed sequences, localized in the deep posterior-lateral cortical area of both cerebellar hemispheres. These findings were compatible with severe post-anoxic damage, thus the patient received two doses of Eculizumab (300 mg IV) on day 4 and 11.

The patient remained in PICU for 14 days and was also treated with the best supportive therapy, including also IV antibiotics to treat a septic condition (culture-positive bronchial washing for $S$. Marcescens).

The general condition progressively improved and laboratory parameters gained almost normal values within 18 days. A further bMR imaging, on day 27, revealed a significant improvement of the ischemic hypoxic area at cerebellar level, confirmed by normal electrical activity at EEG monitoring.

After extubating, the neurological assessment revealed marked bilateral motor deficit with hyporeflexia/areflexia in the lower limbs and hyperextension of the thigh, while the upper limbs presented intentional tremors and normal reflexes. Moreover, the child also exhibited head postural tremors.

The patient returned to our Nephrology Unit on day 16. At admission, the diuresis was adequate and the renal function was recovered $(\mathrm{s} C \mathrm{Cr} 1.06 \mathrm{mg} / \mathrm{dL})$. Hypokalemia resulting from brisk diuresis was treated with intravenous potassium replacement until it normalized, while blood pressure control was improved with amlodipine and ramipril.

Due to the persistent severe bilateral motor deficit with hyporeflexia/areflexia in the lower limbs and intentional tremors of both the upper limbs, a deep neurological reassessment with ENoG revealed severe axonal damage with both motor and sensory demyelination of the lower limbs, mainly at right. The peripheral polyneuropathy was treated with therapeutic plasma exchange (TPE) (seven treatments), but the functional recovery was unsatisfactory, because of the persisting of lower limb asthenia and bilateral absence of osteotendinous reflexes. Thus, on day 35 the patient was discharged and was transferred to a neuro-rehabilitation center for intensive treatment. Main clinical and therapeutic information about this case is reported as a timeline in Figure 3.

\section{Neuro-rehabilitation program}

Before starting the treatment, neurological examination and physiatric functional evaluation in both patients revealed an inability to walk and symmetrical hypotonia, with lower limbs weaker than upper limbs, lying in a frog-like position, deep tendon reflexes decreased in all extremities. No Babinski's 
bilateral signs were present. Furthermore, patient 1 showed distal lower limbs mild hypotrophy and scoliosis, while patient 2 clinical presentation was more severe, with bilateral ankle contractures. The neuropsychological profile was within normal limits in both patients.

In patient 1, visual evoked potential showed both the reductions in P100 amplitude and increments in P100 latency at the right occipital area, while no specific abnormalities have been revealed on the left. The latter was normal in patient 2, who instead presented retinal dystrophy in the right eye and scotopic and photopic full-field ERG characterized by reduced amplitudes particularly in scotopic condition; the left eye was normal.

In these cases, physiotherapy and occupational therapy were started including specific therapeutic activities; during the first stage, when the voluntary movement was compromised, passive kinesitherapy has been initiated to improve local blood circulation and to avoid joint stiffness, tendon retractions, and muscle contractures due to protracted immobility.

After the appearance of voluntary activity event, active kinesitherapy has been progressively introduced through a gentle submaximal and aerobic functional exercise, that was carried out with adequate monitoring, with pauses according to the needs of the patient and with graduated assistance $\left({ }^{21}\right)$.

In patient 2, ankle foot orthoses (AFOs) was also used to minimize contractures and to improve both the stance and swing phases of gait.

Moreover, the neuro-rehabilitation program included exercises to improve coordination and balance. Our patients received this specific and intensive physiotherapy program and, after a two months term, they developed a progressive improvement on muscular strength and on motor functions.

We have used the Functional Independence Measure for Children (WeeFIM) $\left({ }^{22}\right)$ to assess outcomes from neuro-rehabilitation and significant changes have been found in both patients particularly concerning to the motor scale (Table 1).

In both patients, neurological examination revealed the recovery of autonomous deambulation and improvement in postural changes, but the walk was still unstable, with a wide base and the feet thrown out, and the equilibrium was mildly unstable. Besides, both children showed full adherence to the hospital-based neuro-rehabilitation program without significant adverse events, and they continued a home-based rehabilitation once discharged. After further 8 months of home rehabilitation, both patients were re-evaluated with evidence of complete functional recovery.

\section{Genetic screening}

Both the patients were clearly affected by a STEC-HUS related to E. Coli 0111 infection. However, due to very severe neurological impairment, genetic screening of all exons and flanking regions of $\mathrm{CFH}, \mathrm{CD} 46$, CFI, CFB, C3, THBD, DGKE, C5, CFHR1-5, MMACHC and ADAMTS13 was performed through nextgeneration sequencing (NGS), as described elsewhere $\left({ }^{23},{ }^{24}\right)$, but no pathogenic variants were identified. 
Moreover, none of them presented genetic variants of C5 gene, which are related to low response to Eculizumab $\left({ }^{25}\right)$.

\section{Discussion And Conclusion}

Neurological involvement is the most frequent extra-renal manifestation during STEC-HUS, accounting for almost $17-34 \%$ of patients (7). This complication significantly worsens patients' survival and may lead to severe permanent disability, since end stage renal disease requiring chronic hemodialysis and/or kidney transplantation following STEC-HUS-induced AKI accounts for only $3 \%$ of cases $(26,27,28)$.

STEC-HUS-mediated CNS injury may vary from mild to severe and potentially life-threatening manifestations, ranging from eye disorders (strabismus/eye fixing, nystagmus, visus disorders/amaurosis), to alterations of communication skills, to CNS disorders (hypo/hypertensive disorders of muscle tone, neuro-vegetative system disorders, alteration of consciousness, epileptic seizures until coma) (7).

Among neurological assessment techniques, brain MR is actually the gold standard method to analyze brain damage location and extent during STEC-HUS, which mainly, but not only, involves basal nuclei with a pattern in early diffusion-weighted T1 sequence. However, given the wide clinical spectrum of neurological signs, lesions at different levels of CNS have also been reported $\left({ }^{29}\right)$. Based on both MR findings and the location of the lesions, the main pathogenic mechanism seems to be ischemic injury after thrombotic microangiopathy onset with rising evidence of complement system involvement $\left({ }^{30}\right)$. Although controlled clinical trials are lacking, all these findings strongly suggest a key role of early administration of Eculizumab, and anti-C5 convertase monoclonal antibody, to halt and potentially reverse the TMA-mediated severe neurological involvement, as well as at renal levels. Rising evidence support the efficacy and safety of Eculizumab administration to treat the severe neurologic involvement in STEC-HUS patients $\left({ }^{31}, 32\right)$. Indeed, even in our cases, the central nervous system damage following STEC-HUS onset was effectively reverted by early Eculizumab administration.

Although several works describe a wide spectrum of clinical and radiological signs affecting CNS during STEC-HUS, very little is reported about peripheral nerve involvement. In a single report, the authors described the case of a pediatric patient with severe STEC-HUS belatedly complicated by a disabling peripheral neuropathy, as in our two cases (11).

Neurological emergencies are frequent in patients with hematological disorders, for instance during thrombotic microangiopathies, which involves both the central and peripheral nervous system $\left({ }^{33}\right)$. In limited cases, when nerve biopsy was available, thrombosis of epineurial blood vessels with inflammation was shown, thus demonstrating that thrombotic microangiopathy can involve the peripheral nerves, resulting in major morbidity $\left({ }^{34}\right)$. In this report, we described two cases of severe PNS involvement following STEC-HUS infection and underline the efficacy of a prompt neuro-rehabilitation program, coupled with adequate supportive care. The pathogenic mechanism of peripheral nerve 
involvement during STEC-HUS is unknown. In experimental models, the evidence of verotoxin receptors in peripheral nerves strongly suggests a possible direct neuropathic effect of STEC infection $\left({ }^{35}\right)$. However, in our two cases, the lack of nerve biopsy does not allow us to draw definitive conclusions about the pathogenesis of the STEC-HUS-related peripheral neuropathy. Intriguingly in both cases, the late onset of PNS involvement after resolution of CNS injury seems to suggest a possible role for dystrophic phenomena affecting the peripheral nerves, probably due to STEC-HUS-related lack of nutrients (i.e. probiotics or vitamins) $\left.{ }^{36}\right)$, which in other clinical settings may lead to peripheral neuropathy $(37,38)$. Generally, we can consider the peripheral nerve as a complex system in which the endoneurial homeostasis is strictly regulated via tight junction-forming endoneurial microvessels that control ion, solute, water, nutrient, macromolecule, and leukocyte influx and efflux between the bloodstream and endoneurium; thus, it is not daring to speculate that the STEC-related blood stream disorders (i.e. leukocytosis, electrolyte alterations, toxin trafficking) may affect the blood-nerve barrier integrity and function, thus leading to more or less severe decay of the peripheral nervous system $\left({ }^{39}\right)$.

Although immunotherapies with monoclonal antibodies may induce peripheral neuropathic side effects and, rarely, immune axonal acquired neuropathies $\left({ }^{40}\right)$, this complication seems unlikely after Eculizumab infusion and up to now no cases have been described. Moreover, in the second case here reported, the severe peripheral neuropathy could be immune-mediated, thus suggesting a rescue therapy with plasmaexchange (PE). Unlike rehabilitation therapy, this approach did not gain any benefit and was halted after 6 sessions. The absent response to PE further excludes the hypothesis of an immune-mediated peripheral neurological damage in our cases.

In conclusion, peripheral nerve involvement during STEC-HUS is a very rare, but potentially severely invalidating event. Although we described just two cases of severe PNS involvement following STEC-HUS infection, the early treatment of this complication with prompt neuro-rehabilitation, coupled with adequate supportive care, is essential to prevent permanent and irreversible damage and may significantly improve the clinical outcome of these pediatric patients.

\section{List Of Abbreviations}

AFO

aHUS

BE

CFE:

CMAP

CNS:

CPR:
Ankle Foot Orthoses

atypical Hemolytic uremic syndrome

Base Excess

Cerebrospinal fluid examination

Compound Motor Action Potentials

Central nervous system

Cardiopulmonary resuscitation 
CRP:

C-reactive protein

CT

Computer Tomography

EEG:

Electroencephalography

EHEC:

Entero-Hemorragic Escherichia Coli

EMG

Electromyography

ENoG

Electroneurography

ERG

Electroretinography

FIM

Functional Independence Measure

GCS:

Glascow Coma Score

$\mathrm{HCO}^{3-}:$

Bicarbonate Ion

HDF:

Hemodialfiltration

HGB:

Haemoglobin

HUS:

Hemolytic uremic syndrome

IV:

Intra-venous (therapy)

LDH:

Lactate dehydrogenase

MR:

Magnetic resonance

Neut:

Neutrophils

PICU:

Pediatric intensive care unit

PLT:

Platelets (count)

PNS:

Peripheral nervous system

RRT:

Renal replacement therapy

SAP

Sensory Action Potential

$\mathrm{sCr}$ :

serum Creatinine

STEC:

Shiga-like toxin-producing Escherichia coli 
WBC:

White blood cell (count)

\section{Declarations}

\section{Ethics approval and consent to participate}

The present study has been carried out retrospectively based on registry data. All procedures performed were in accordance with the 1964 Helsinki declaration and its later amendments. Ethical approval was obtained from the Institutional Review Board of the University Hospital "Policlinico Consorziale" of Bari (Italy) (Prot. 1624/2018).

\section{Consent for publication}

The authors declare their consent to publication this manuscript in the present form.

All the minors' legal guardians signed a written informed consent to collect their clinical data at time of hospital access and for the publication of any potentially identifiable images or data included in this article.

\section{Availability of data and materials}

The datasets used and/or analysed during the current study are available from the corresponding author on reasonable request.

\section{Competing interests}

The authors declare that they have no competing interests.

\section{Funding}

The present work was funded by University Research Projects 2019 (PRA 2019) granted by University of Foggia to Giuseppe Stefano Netti.

\section{Authors' contributions}

Luisa Santangelo conceived and designed the study, analyzed the data and drafted the manuscript; Giuseppe Stefano Netti analyzed the data, interpreted results and prepared the figures; Diletta D. Torres, Giovanni Piscopo, Vincenza Carbone, Luciana Losito, Leonardo Milella, Maria Luigia Lasorella, Pasquale Conti and Delio Gagliardi collected the clinical data and helped to interpret the results; Maria Chironna, Elena Bresin and Antonio Trabacca, helped to draft the manuscript; Elena Ranieri and Mario Giordano edited and revised manuscript and approved the final version of manuscript. 
Acknowledgements

The authors thank Mrs. Mariella Ragone, the nurse coordinator, and Dr. Antonio Ranieri and Vito Bellino, Dialysis Technician at the Pediatric Nephrology Unit of the Pediatric Hospital "Giovanni XXIII", Bari (Italy), for their invaluable collaboration.

Authors' information (optional)

Pediatric Nephrology and Dialysis Unit, Pediatric Hospital 'Giovanni XXIII', Bari (Italy)

Luisa Santangelo, Diletta Domenica Torres, Giovanni Piscopo, Vincenza Carbone, Mario Giordano Clinical Pathology Unit and Center for Molecular Medicine, Department of Medical and Surgical Sciences, University of Foggia, Foggia (Italy)

Giuseppe Stefano Netti, Elena Ranieri

Scientific Institute I.R.C.C.S. "E. Medea"- Unit for Severe disabilities in developmental age and young adults (Developmental Neurology and Neurorehabilitation), Brindisi (Italy)

Luciana Losito, Antonio Trabacca

Intensive Care Unit, Pediatric Hospital "Giovanni XXIII", Bari (Italy)

Leonardo Milella

Pediatric Neurology Unit, Pediatric Hospital "Giovanni XXIII", Bari (Italy)

Maria Luigia Lasorella, Pasquale Conti, Delio Gagliardi

Department of Biomedical Sciences and Human Oncology, Hygiene Unit, University of Bari "Aldo Moro", Bari (Italy)

Maria Chironna

Clinical Research Center for Rare Diseases 'Aldo e Cele Daccò', Istituto di Ricerche Farmacologiche Mario Negri IRCCS, Bergamo (Italy)

Elena Bresin

\section{References}

1. Mele C, Remuzzi G, Noris M. Hemolytic uremic syndrome. Semin Immunopathol. 2014; 36:399-420. doi:10.1007/s00281-014-0416-x 
2. Geerdink LM,Westra D, vanWijk JA, Dorresteijn EM, Lilien MR,Davin JC, et al. Atypical hemolytic uremic syndromein children: complement mutations and clinical characteristics. Pediatr Nephrol. 2012; 27:12831291. doi: 10.1007/s00467-012-2131-y

3. Noris M, Caprioli J, Bresin E, Mossali C, Pianetti G, Gamba S, et al. Relative role of genetic complement abnormalitiesin sporadic and familial aHUS and their impact on clinicalphenotype. Clin J Am Soc Nephrol. 2010; 5:1844-1859. doi: 10.2215/CJN.02210310

4. Fremeaux-Bacchi V, Fakhouri F, Garnier A, Bienaime F, Dragon-Durey MA, et al. Genetics and outcome of atypical hemolytic uremic syndrome:a nationwide French series comparing children and adults. Clin $\mathrm{J}$ Am Soc Nephrol. 2013; 8:554-562. doi: 10.2215/CJN.04760512

5. Jokiranta TS. HUS and atypical HUS. Blood. 2017; 129(21):2847-2856. doi: 10.1182/blood-2016-11709865.

6. Loirat C, Fakhouri F, Ariceta G, Besbas N, Bitzan M, Bjerre A, et al. An international consensus approach to the management of atypical hemolytic uremic syndrome in children. Pediatr Nephrol. 2016; 31(1):1539. doi: 10.1007/s00467-015-3076-8

7. Khalid M, Andreoli S. Extrarenal manifestations of the hemolytic uremic syndrome associated with Shiga toxin-producing Escherichia coli (STEC HUS). Pediatr Nephrol. 2019; 34:2495-2507. doi:10.1007/s00467-018-4105-1

8 . Matthies J, Hünseler C, Ehren R, Volland R, Körber F, Hoppe B, et al. Extrarenal Manifestations in Shigatoxin-Associated Haemolytic Uremic Syndrome. Klin Paediatr. 2016; 228:181-188. doi: 10.1055/s0042-108444

9. Trachtman H, Austin C, Lewinski M, Stahl RA. Renal and neurological involvement in typical Shiga toxin-associated HUS. Nat Rev Nephrol. 2012; 8:658-669. doi: 10.1038/nrneph.2012.196

10. Otukesh H, Hoseini R, Golnari P, Fereshtehnejad SM, Zamanfar D, Hooman N, et al. Short-term andlong-term outcome of hemolytic uremic syndrome inlranian children. J Nephrol. 2008; 21:694-703. PMID: 18949724

11 . Santangelo L, Netti GS, Giordano P, Carbone V, Martino M, Torres DD, et al. Indications and results of renal biopsy in children: a 36-year experience. World J Pediatr. 2018; 14(2):127-133. doi: 10.1007/s12519018-0147-5

12. Adamczuk D, Ziołkowska H, Leszczyńska B, Roszkowska-Blaim M. Ciezki przebieg typowego zespołu hemolityczno-mocznicowego u 14-letniego chłopca [Severe course of typical hemolytic-uremic syndrome in a 14-year-old boy]. Pol Merkur Lekarski. 2009; 26:340-342. PMID: 19580203

13. Thurman JM, Marians R, Emlen W, Wood S, Smith C, Akana H et al. Alternative pathway of complement in children with diarrhea-associated hemolytic uremic syndrome. Clin J AmSocNephrol. 
14. Lapeyraque AL, Malina M, Fremeaux-Bacchi V, Boppel T, Kirschfink M, Oualha M, et al. Eculizumab in severe Shiga-toxin-associated HUS. N Engl J Med. 2011; 364:2561-2563. doi: 10.1056/NEJMc1100859

15. Giordano P, Netti GS, Santangelo L, Castellano G, Carbone V, Torres DD, et al. A pediatric neurologic assessment score may drive the eculizumab-based treatment of Escherichia coli-related hemolytic uremic syndrome with neurological involvement. Pediatr Nephrol. 2019; 34:517-527. doi: 10.1007/s00467-0184112-2

16. Netti GS, Santangelo L, Paulucci L, Piscopo G, Torres DD, Carbone V, et al. Low C3 Serum Levels Predict Severe Forms of STEC-HUS With Neurologic Involvement. Front Med (Lausanne) 2020; 7:357. doi: 10.3389/fmed.2020.00357

17. Walsh PR, Johnson S. Eculizumab in the treatment of Shiga toxin haemolytic uraemic syndrome. Pediatr Nephrol. 2019; 34(9):1485-1492. doi: 10.1007/s00467-018-4025-0.

18 . Loconsole D, Giordano M, Laforgia N, Torres DD, Santangelo L, Carbone V, et al. Case-management protocol for bloody diarrhea as a model to reduce the clinical impact of Shiga toxin-producing Escherichia coli infections. Experience from Southern Italy. Eur J Clin Microbiol Infect Dis. 2019; 39:539547. doi:10.1007/s10096-019-03755-0

19. Netti GS, Sangregorio F, Spadaccino F, Staffieri F, Crovace A, Infante B, et al. LPS removal reduces CD80-mediated albuminuria in critically ill patients with Gram-negative sepsis. Am J Physiol Renal Physiol. 2019; 316(4):F723-F731. doi: 10.1152/ajprenal.00491.2018

20. Castellano G, Stasi A, Franzin R, Sallustio F, Divella C, Spinelli A, et al. LPS-Binding Protein Modulates Acute Renal Fibrosis by Inducing Pericyte-to-Myofibroblast Trans-Differentiation through TLR-4 Signaling. Int J Mol Sci. 2019; 20(15):3682. doi: 10.3390/ijms20153682

21. Casale R, La Manna A, Salvini S, Maini M, Ceccherelli F, Frazzitta G. Peripheral neuropathy rehabilitation. Indications for a diagnostic-rehabilitation approach. G Ital Med Lav Ergon. 2003; 25:45664. PMID: 15027694

22 . Ottenbacher KJ, Msall ME, Lyon N, Duffy LC, Ziviani J, CGranger CV, et al. The WeeFIM instrument: Its utility in detecting change in children with developmental disabilities. Archives of Physical Medicine and Rehabilitation. 2000; 81:1317-1326. doi: 10.1053/apmr.2000.9387

23. Valoti E, Alberti M, latropoulos P, Piras R, Mele C, Breno M, et al. Rare Functional Variants in Complement Genes and Anti-FH Autoantibodies-Associated aHUS. Front Immunol. 2019; 10:853. doi: 10.3389/fimmu.2019.00853.

24. de Martino M, Gigante M, Cormio L, Prattichizzo C, Cavalcanti E, Gigante M, et al. JAK3 in clear cell renal cell carcinoma: mutational screening and clinical implications. Urol Oncol. 2013; 31(6):930-937. doi: 
25 . Nishimura J, Yamamoto M, Hayashi S, Ohyashiki K, Ando K, Brodsky AL, et al. Genetic variants in C5 and poor response to eculizumab. N Engl J Med. 2014; 632-9. doi: 10.1056/NEJMoa1311084.

26. Spinale JM, Ruebner RL, Copelovitch L, Kaplan BS. Long-term outcomes of Shiga toxin hemolytic uremic syndrome. Pediatr Nephrol. 2013; 28:20972105. doi: 10.1007/s00467-012-2383-6

27. Santangelo L, Gigante M, Netti GS, Diella S, Puteo F, Carbone V, et al. A novel SMARCAL1 mutation associated with a mild phenotype of Schimke immuno-osseous dysplasia (SIOD). BMC Nephrol. 2014; 15:41. doi:10.1186/1471-2369-15-41

28. Gigante M, d'Altilia M, Montemurno E, Diella S, Bruno F, Netti GS, et al. Branchio-Oto-Renal Syndrome (BOR) associated with focal glomerulosclerosis in a patient with a novel EYA1 splice site mutation. BMC Nephrol. 2013; 14:60. doi:10.1186/1471-2369-14-60

29. Löbel U, Eckert B, Simova O, Meier-Cillien M, Kluge S, Gerloff C, et al. Cerebral magnetic resonance imaging findings in adults with haemolytic uraemic syndrome following an infection with Escherichia coli, subtype 0104:H4. Clin Neuroradiol. 2014; 24:111-119. doi:10.1007/s00062-013-0231-0

30 . Trachtman H, Austin C, Lewinski M, Stahl RA. Renal and neurological involvement in typical Shiga toxin-associated HUS. Nat Rev Nephrol. 2012; 8:658-669. doi:10.1038/nrneph.2012.196

31. Pape L, Hartmann H, Bange FC, Suerbaum S, Bueltmann E, Ahlenstiel-Grunow T. Eculizumab in Typical Hemolytic Uremic Syndrome (HUS) With Neurological Involvement. Medicine (Baltimore). 2015; 94:e1000. doi: 10.1097/MD.0000000000001000

32. Percheron L, Gramada R, Tellier S, Salomon R, Harambat J, Llanas B, et al. Eculizumab treatment in severe pediatric STEC-HUS: a multicenter retrospective study. Pediatr Nephrol. 2018; 33:1385-1394. doi: 10.1007/s00467-018-3903-9

33. Cruz ML, Farooq S, Testai FD. Neurological and Neurosurgical Emergencies in Patients with Hematological Disorders. Curr Neurol Neurosci Rep. 2017; 17:24. doi: 10.1007/s11910-017-0728-z

34 . Naddaf E, James B Dyck P, Said S, Amer H. Thrombotic microangiopathy involving kidney allograft and peripheral nerves. Am J Transplant. 2020; 20:1454-1456. doi: 10.1111/ajt.15736

35. Ren J, Utsunomiya I, Taguchi K, Ariga T, Tai T, Ihara Y, et al. Localization of verotoxin receptors in nervous system. Brain Res. 1999; 825:183188. doi: 10.1016/s0006-8993(99)01196-8

36. Giordano M, Baldassarre ME, Palmieri V, Torres DD, Carbone V, Santangelo L, et al. Management of STEC Gastroenteritis: Is There a Role for Probiotics?. Int J Environ Res Public Health. 2019; 16:1649. doi: 10.3390/ijerph16091649 
37. Nadjar Y, Souvannanorath S, Maisonobe T, Brisset M, De Lonlay P, Schiff M,et al. Sensory neuronopathy as a major clinical feature of mitochondrial trifunctional protein deficiency in adults. Rev Neurol (Paris) 2020; 176:380-386. doi: 10.1016/j.neurol.2019.11.011

38 . Morel L, Domingues O, Zimmer J, Michel T. Revisiting the Role of Neurotrophic Factors in Inflammation. Cells. 2020; 9:E865. doi:10.3390/cells9040865

39. Ubogu EE. Biology of the human blood-nerve barrier in health and disease. Exp Neurol. 2020; 328:113272. doi:10.1016/j.expneurol.2020.113272

40 . Davies AJ, Rinaldi S, Costigan M, Oh SB. Cytotoxic Immunity in Peripheral Nerve Injury and Pain. Front Neurosci. 2020; 14:142. doi:10.3389/fnins.2020.00142

\section{Table}

Table 1. Overview of the Functional Independence Measure (FIM) for children at admission and discharge

\begin{tabular}{lccccc} 
Case 1 & \multicolumn{5}{c}{ Case 2 } \\
\hline Item (scale) & Admission & Discharge & Item (scale) & Admission & Discharge \\
\hline FIM motor & 2.08 & 4.62 & FIM motor & 1.23 & 1.69 \\
\hline FIM cognitive & 3.22 & 5.11 & FIM cognitive & 2.6 & 2.6 \\
\hline & & & & & \\
FIM total & 58 & 92 & FIM total & 29 & 35
\end{tabular}

\section{Figures}


A

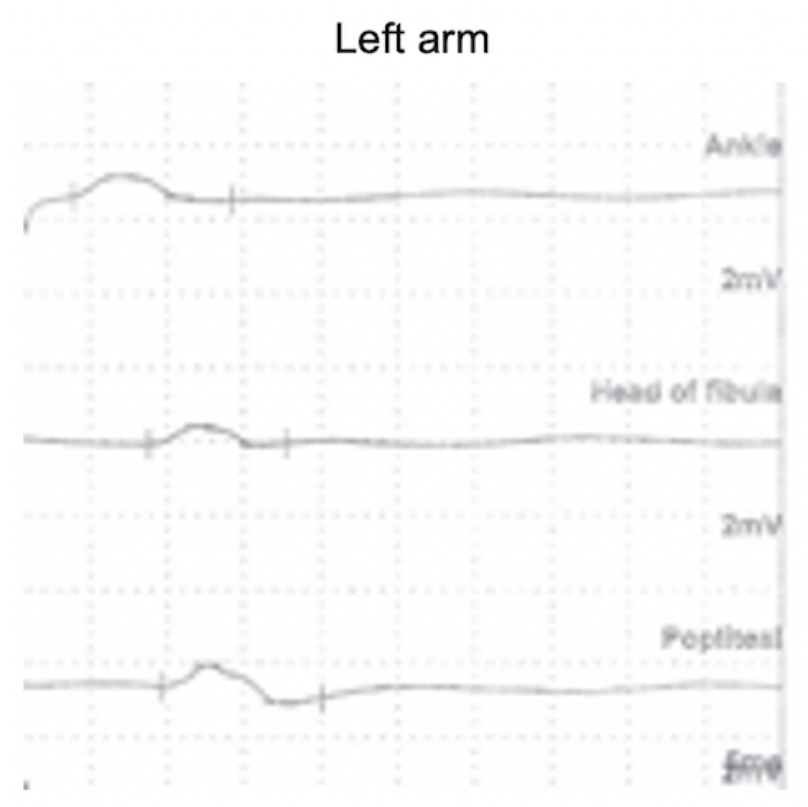

Right arm

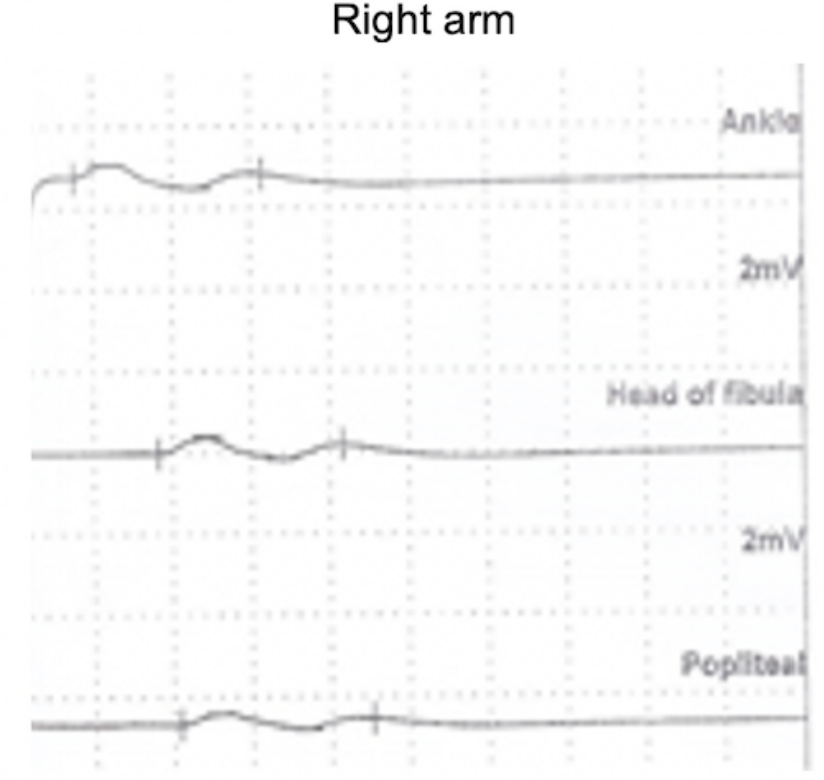

B

Left leg

Right leg
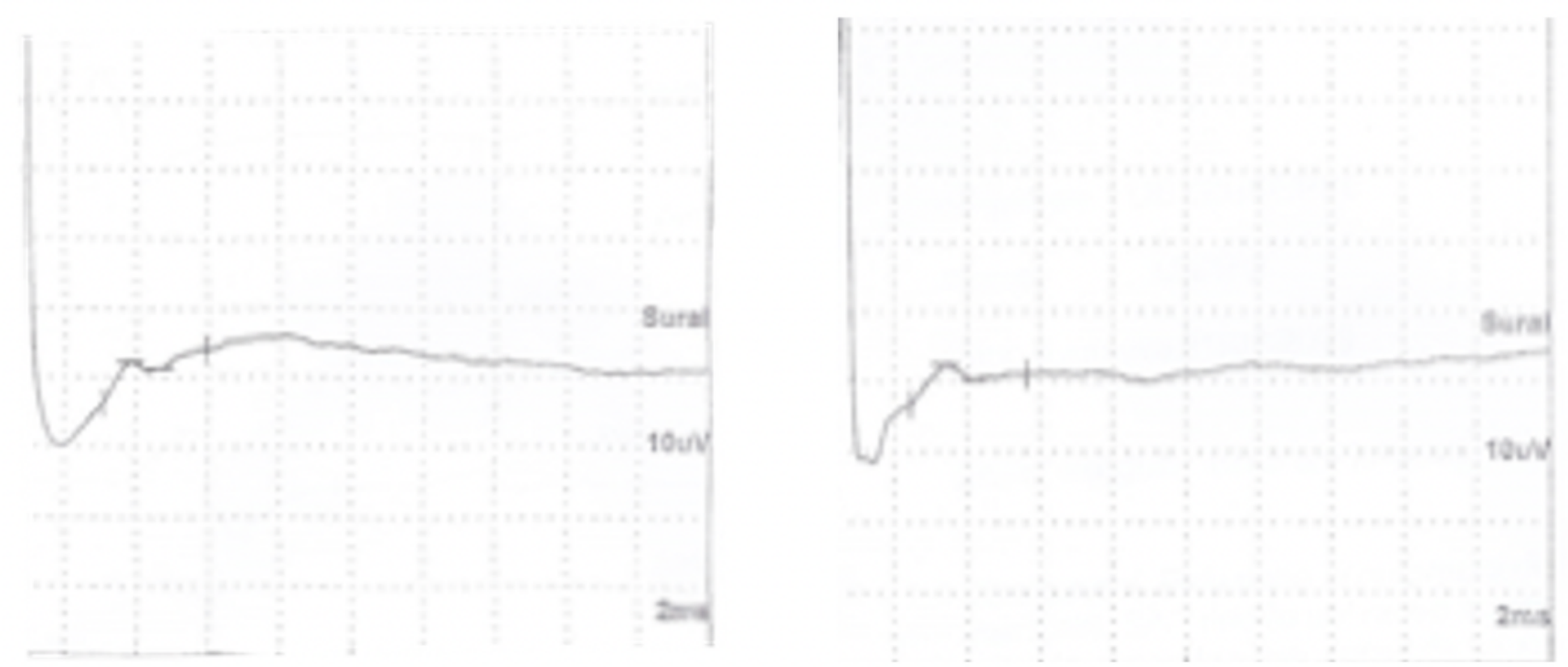

\section{Figure 1}

Electroneurography of peripheral nerves revealing severe polineuropathy Low amplitude of the compound motor action potentials (CMAP) in three sites at the peroneal motor nerves (A) and reduction of sensory action potential (SAP) at sural sensory nerves, assessed bilaterally by electroneurography (ENoG) of peripheral nerves in patient 1. 

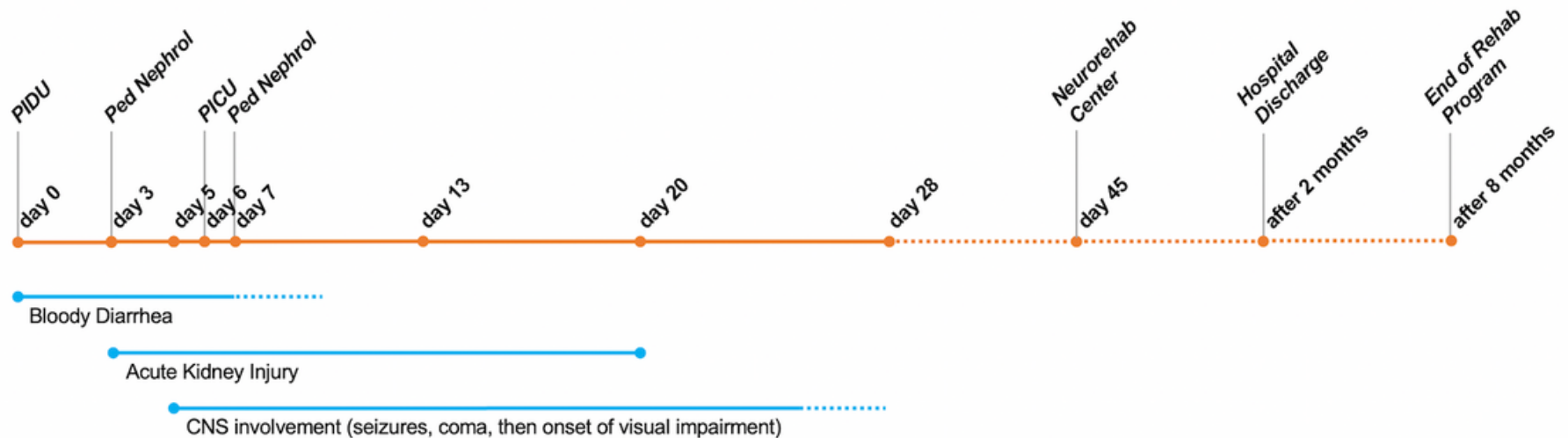

CNS involvement (seizures, coma, then onset of visual impairment)
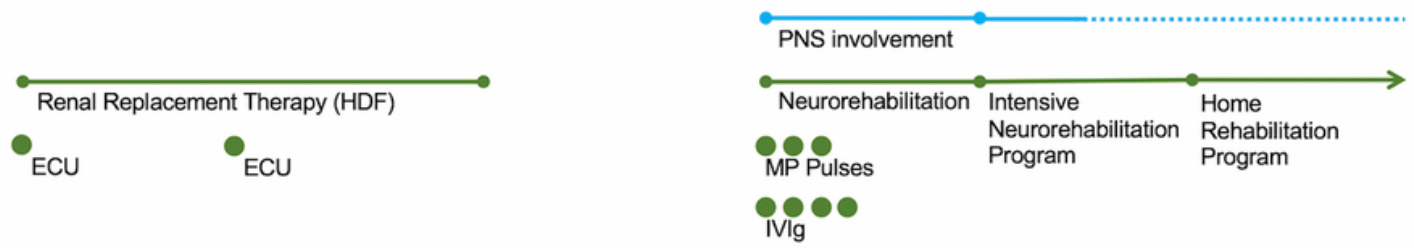

Figure 2

Timeline of Patient 1 CNS, central nervous system; ECU, eculizumab; IVIG, intravenous immunoglobulin; MP, methylprednisolone; PNS, peripheral nervous system.
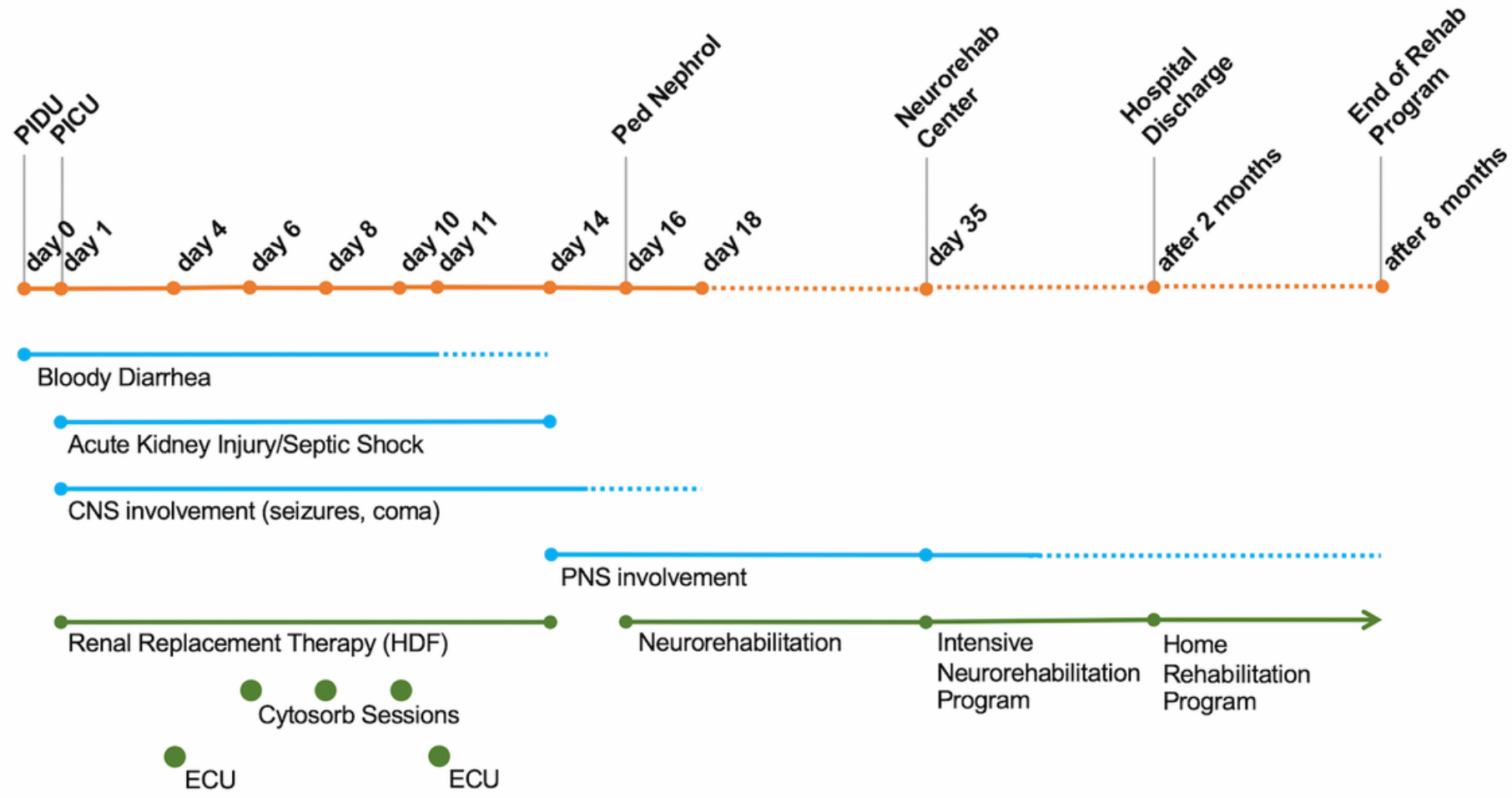

PNS involvement

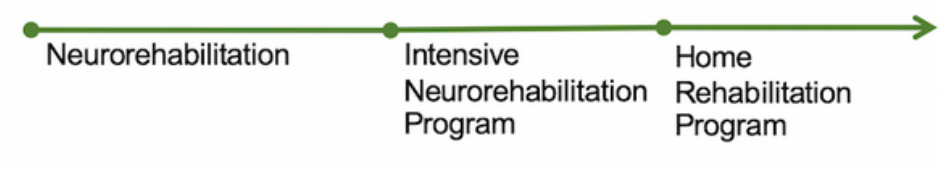

\section{Figure 3}

Timeline of Patient 1 CNS, central nervous system; ECU, eculizumab; PNS, peripheral nervous system; TPE, Therapeutic Plasma Exchange. 


\section{Supplementary Files}

This is a list of supplementary files associated with this preprint. Click to download.

- CaseReportCAREchecklist.docx 